\title{
Resistance to epithelial growth factor receptor tyrosine kinase inhibitors in a patient with transformation from lung adenocarcinoma to small cell lung cancer: A case report
}

\author{
LIYING FANG $^{1,2^{*}}$, JIAN HE $^{1 *}$, JINGWEN XIA $^{1}$, LIANG DONG $^{1}$, XIUJUAN ZHANG ${ }^{1}$, YAQIN CHAI $^{1,3}$, \\ YING LI ${ }^{1,4}$, MENGJIE NIU ${ }^{1}$, TIANXING HANG ${ }^{1}$ and SHENGQING LI ${ }^{1}$ \\ ${ }^{1}$ Department of Respiratory Medicine, Huashan Hospital, Fudan University, Shanghai 200040; \\ ${ }^{2}$ Department of Respiratory Medicine, The Fourth People's Hospital of Shaanxi, Xi'an, Shaanxi 710043; \\ ${ }^{3}$ Department of Respiratory Medicine, Xi'an Aerospace General Hospital, Xi'an, Shaanxi 710100; \\ ${ }^{4}$ Department of Respiratory Medicine, The Second People's Hospital of Shaanxi, Xi'an, Shaanxi 710005, P.R. China
}

Received June 1, 2016; Accepted February 23, 2017

DOI: $10.3892 / \mathrm{ol} .2017 .6229$

\begin{abstract}
First-generation epithelial growth factor receptor tyrosine kinase inhibitors (EGFR-TKIs) have markedly improved the treatment of non-small cell lung cancer (non-SCLC) with EGFR-sensitive mutations. However, acquired resistance to these drugs was inevitable. The transformation of lung adenocarcinoma to SCLC following treatment with EGFR-TKIs is a rare phenomenon that contributes to resistance to EGFR-TKIs. The present case concerns a 74-year-old man previously diagnosed with and treated for pneumonia; however, this was later pathologically confirmed as lung adenocarcinoma by transbronchial lung biopsy. Deletion of exon 19 of EGFR was identified by next-generation sequencing technology. The patient improved markedly when treated with gefitinib, but relapsed after 1 year, with markedly increased serum levels of neuron-specific enolase (NSE). Transformation to SCLC was detected by endobronchial ultrasound transbronchial needle aspiration (EBUS-TBNA) re-biopsy, which was negative for the deletion of exon 19 of EGFR. The patient was positive for vimentin expression and refractory to etoposide and cisplatin chemotherapy, and succumbed to the disease 18 months after diagnosis. Transformation of the disease from adenocarcinoma to SCLC may have been due to cancer heterogeneity. Re-biopsy is therefore important in EGFR-TKI-resistant patients for genetic and
\end{abstract}

Correspondence to: Professor Shengqing Li, Department of Respiratory Medicine, Huashan Hospital, Fudan University, 12 Urumqi Middle Road, Shanghai 200040, P.R. China

E-mail: shengqingli@hotmail.com

*Contributed equally

Key words: epithelial growth factor receptor tyrosine kinase inhibitor, lung adenocarcinoma, small cell lung cancer, resistance histological re-evaluation. NSE serum levels may also be useful for detecting early SCLC transformation.

\section{Introduction}

Lung cancer, particularly non-small-cell lung cancer (NSCLC), remains the leading cause of global cancer-associated mortality, with a 5-year survival rate of $<20 \%$ (1). Despite years of research into novel chemotherapy combinations, few treatment options are available for the majority of patients with advanced or metastatic disease (2). Adenocarcinoma patients with epithelial growth factor receptor tyrosine kinase inhibitor (EGFR-TKI)-sensitive mutations generally exhibit improved progression-free and overall survival times following TKI treatment (3). However, these patients inevitably encounter resistance to first-generation EGFR-TKIs, usually after 6-8 months of treatment (4). There are several mechanisms involved in the generation of EGFR-TKI resistance in non-small cell lung cancer (NSCLC) (5). These include the T790M EGFR mutation, which induces secondary resistance in $>50 \%$ of patients (6); the compensatory contribution of other receptor tyrosine kinases, including c-MET amplification (7) and activating mutations in human epidermal growth factor receptor 2 (HER-2) (8); the activation of compensatory signaling pathways, including the phosphoinositide-3 kinase/AKT/mammalian target of rapamycin signaling pathway (9) and the TOPK-c-Jun pathway (10); and histological transformation, including EMT phenotypic transforming (11) and SCLC transformation (12). The transformation of lung adenocarcinoma to SCLC, as described by the present case report, is a relatively rare TKI resistance mechanism.

\section{Case report}

A 74-year-old male with a 50-pack-year smoking history presented to the Department of Respiratory Medicine (Huashan Hospital, Shanghai, China) with a 3-year history of coughing, sputum production and shortness of breath in March 2014. A computed tomography (CT) scan revealed multiple patchy and 
Table I. Driver gene profile of primary lung adenocarcinoma and secondary small cell lung cancer.

Driver gene

Primary lung adenocarcinoma

Secondary small cell lung cancer

EGFR exon 19 deletion

ALK rearrangement

HER 2 mutations

BRAF V600E mutation

High-level MET amplification or MET exon 14

skipping mutation

RET rearrangements

ROS1 rearrangements

KRAS mutation

$\begin{array}{ll}+ & - \\ - & - \\ - & - \\ - & - \\ - & - \\ - & - \\ - & - \\ - & -\end{array}$

EGFR, epithelial growth factor receptor; ALK, anaplastic lymphoma kinase; HER2, human epidermal growth factor receptor 2; BRAF, B-Raf proto-oncogene, serine/threonine kinase; MET, MET proto-oncogene, receptor tyrosine kinase; RET, ret proto-oncogene; ROS, ROS proto-oncogene 1, receptor tyrosine kinase; KRAS, KRAS proto-oncogene, GTPase.

nodular high-density shadows, and bilateral lung bronchiectasis with consolidation in the left lower lobe (Fig. 1A). The structure of the mediastinum was clear and the lymph nodes were not enlarged (Fig. 1B). The patient was administered ceftazidime and levofloxacin to treat the suspected infection prior to hospitalization. However, the patient did not respond to treatment and the symptoms and CT scan images gradually worsened.

Following admission, adenocarcinoma cells and a Lophomonasblattarum infection were detected by pathological cytology following a bronchoalveolar lavage (BAL) that was performed in the right middle lobe of the lung (Fig. 1C). A subsequent transbronchial lung biopsy was performed on the basal segment of the left lower lobe, which detected well-differentiated adenocarcinoma cells (Fig. 1D). Next-generation sequencing (NGS, Illumina Hiseq 4000; Illumina, Inc., San Diego, CA, USA) was employed to check the 8 driver genes with targeted drugs and it was found that exon 19 of EGFR was deleted (Table I). A positron emission tomography (PET)/CT scan revealed intense uptake of $18 \mathrm{~F}$-fluorodeoxyglucose in the left lower lobe, no hypermetabolic lymph nodes, and no hypermetabolic lesions in the brain, abdomen or bone. The patient was prescribed $500 \mathrm{mg}$ metronidazole once a day for 3 months to cure the L. blattarum infection and $250 \mathrm{mg}$ gefitinib once a day for lung cancer treatment, and was followed up every 2-3 months. The patient's symptoms improved markedly, the serum carcinoembryonic antigen level decreased from $5.52 \mathrm{ng} / \mathrm{ml}$ to within the normal range $(<5 \mathrm{ng} / \mathrm{ml})$ and a CT scan revealed partial remission (Fig. 2).

However, after 1 year of gefitinib treatment, in February 2015, serum levels of neuron-specific enolase (NSE) markedly increased from normal range $(<15 \mathrm{ng} / \mathrm{ml})$ to $50.6 \mathrm{ng} / \mathrm{ml}$, with a CT scan revealing a slightly enlarged left lung hilum (Fig. 2). After a further 3 months of gefitinib treatment, the patient again presented with coughing and shortness of breath. A CT scan revealed a substantially enlarged left lung hilum with left lower lobe bronchial patency (Fig. 2). Another endobronchial ultrasound transbronchial needle aspiration biopsy of the left lower lobe and a set of 4- $\mu$ m-thick paraffin sections were cut and mounted on silanized slides. Sections were incubated for
$60 \mathrm{~min}$ at room temperature with primary antibodies from Abcam (Cambridge, UK): Anti-transcription termination factor 1 (cat no. ab204411; dilution, 1:500), anti-vimentin (cat no. ab92547; dilution, 1:200), anti-anaplastic lymphoma kinase (cat no. ab16770; dilution, 1:100), anti-synaptophysin (cat no. ab32127; dilution, 1:400), anti-leukocyte common antigen (cat no. ab40763; dilution, 1:250), anti-NSE (cat no. ab53025; dilution, 1:100), anti-S100 (cat no. ab868; dilution, 1:100), anti-pan cytokeratin (AE1/AE3; cat no. ab86734; dilution, 1:50), and control staining was performed using isotype rabbit IgG (cat no. 3900) or isotype mouse IgG, (cat no. 5415) (Cell Signaling Technology, Inc., Danvers, MA, USA). Control antibodies diluted to the same concentrationas the primary antibody used for analysis in each case. After washing, sections were incubated for $60 \mathrm{~min}$ at room temperature with horseradish peroxidase polymer (pre-diluted; cat no. ab214879 or ab214880; Abcam) and developed with DAB. Immunohistochemical staining confirmed a histological transformation of the adenocarcinoma to SCLC, and positivity for the expression of transcription termination factor 1 , synaptophysin and vimentin (Fig. 3). However, the deletion of EGFR exon 19 could no longer be detected by NGS (Table I). A chemotherapy regimen was added to the gefitinib treatment: Etoposide $\left(100 \mathrm{mg} / \mathrm{m}^{2}\right.$, days 1-3) plus cisplatin (30 mg/m² , days $1-3)(E P)$. After two cycles of chemotherapy and a 30-Gy curative dose of radiotherapy, the patient showed no improvement, and a CT scan revealed that the left lower lobe was almost totally obstructed by an invasive tumor. As the patient could not tolerate any further chemotherapy, high-frequency electrocoagulation was employed to remove the tumor from the left lower lobe of the lung and a stent was implanted to keep the bronchial lumen patent. The patient was then prescribed the best supportive care and succumbed to the disease 18 months after the initial diagnosis.

Written informed consent was obtained for this case report to be published.

\section{Discussion}

Transformation from lung adenocarcinoma to SCLC following EGFR-TKI treatment is a relatively rare mechanism of 
A

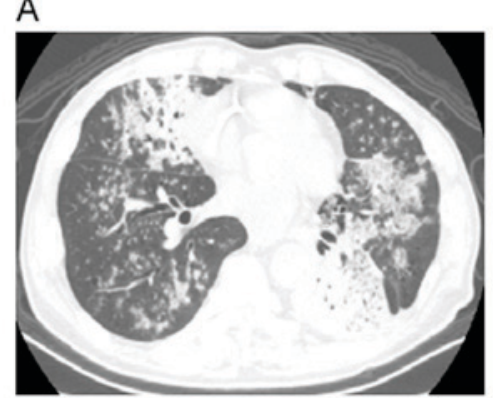

C

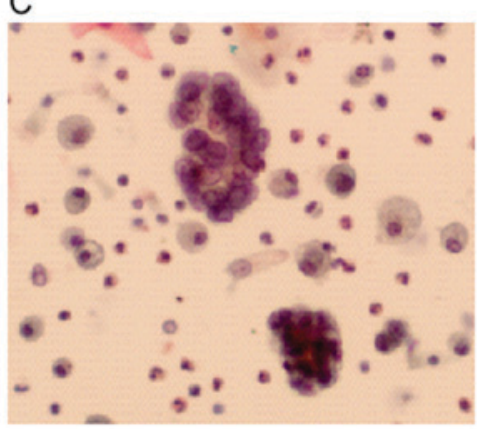

B

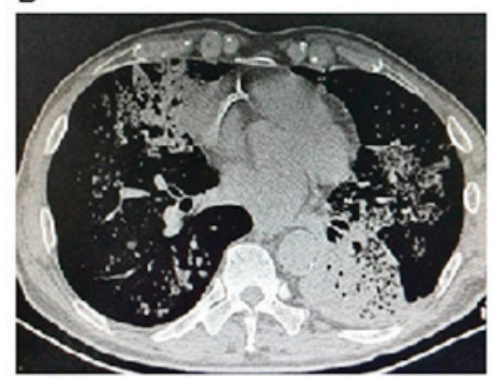

D

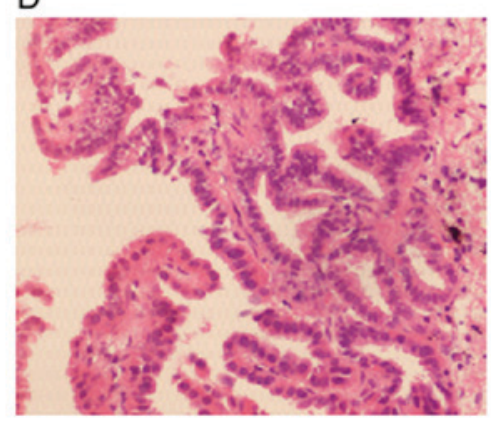

Figure 1. Diagnosis of highly-differentiated lung adenocarcinoma. (A) A CT scan of the pulmonary window revealing multiple patchy and nodular high-density shadows and lung bronchiectasis in the bilateral lungs, and consolidation in the left lower lobe. (B) A CT scan of the mediastinal window showing a clear mediastinal structure without enlarged lymph nodes. (C) Hematoxylin and eosin staining of bronchial alveolar lavage fluid revealing the presence of adenocarcinoma cells and Lophomonasblattarum parasites (magnification, x300). (D) Hematoxylin and eosin staining of a lung biopsy specimen revealing highly-differentiated adenocarcinoma cells (x300 magnification). CT, computed tomography.

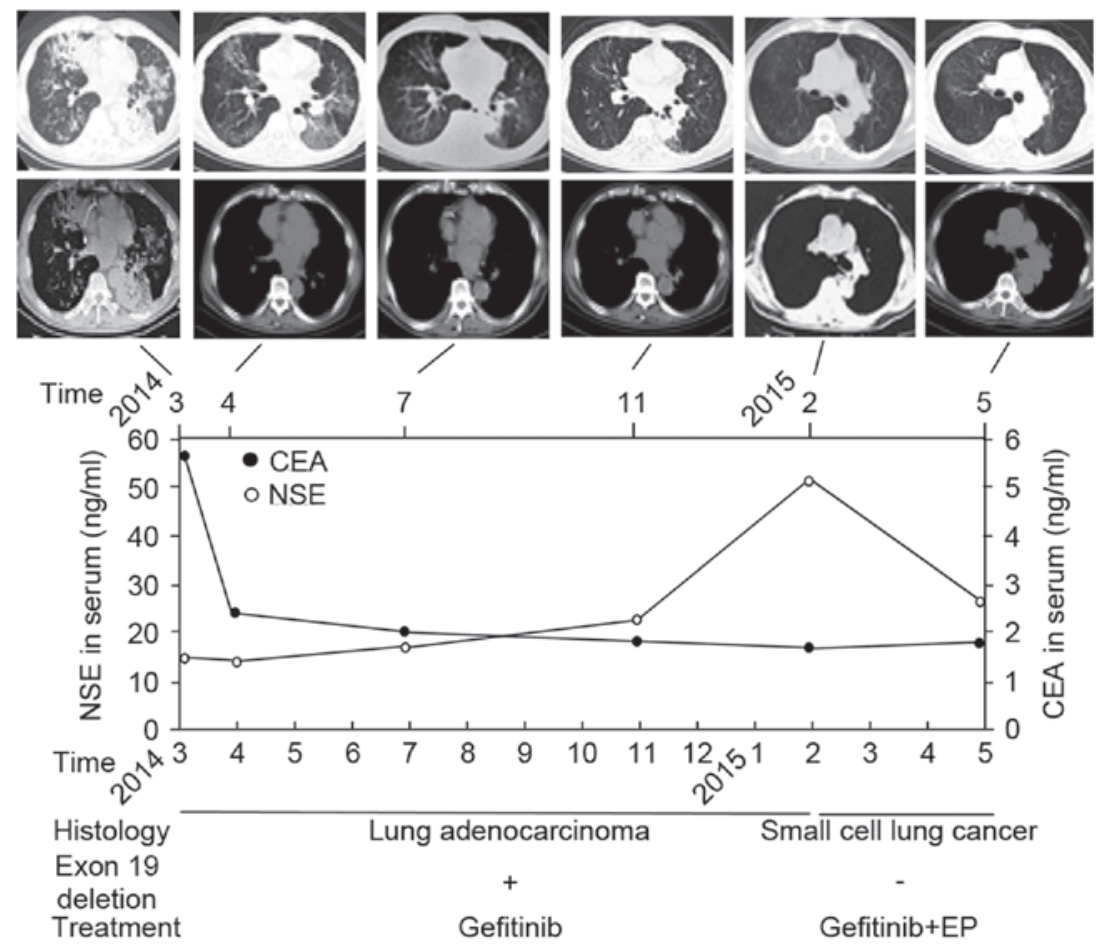

Figure 2. Follow-up schematic diagram. The top panel shows a series of CT scans taken between March 2014 and May 2015; the upper row of images depicts the pulmonary window and the lower row of images depicts the mediastinal window. The middle panel depicts follow-up examinations of serum CEA levels, which decreased markedly after gefitinib treatment, and serum NSE levels, which increased markedly after SCLC transformation. The bottom panel is the pathological diagnosis corresponding to EGFR exon 19 deletion status and the treatment regimen. CT, computed tomography; CEA, carcinoembryonic antigen; NSE, neuron-specific enolase; SCLC, small cell lung cancer; EGFR, epithelial growth factor receptor, EP, etoposide and cisplatin chemotherapy.

resistance to EGFR-TKIs (13). SCLC transformation is more frequent in lung adenocarcinomas that have EGFR-activating mutations than in EGFR wild-type tumors (14). One previous study reported an incidence of SCLC transformation of $3.5 \%$ 


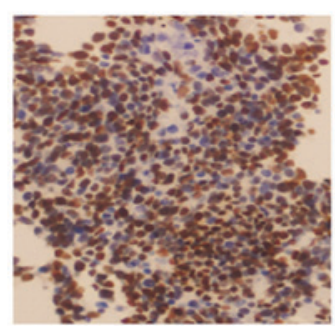

TTF1 (+)

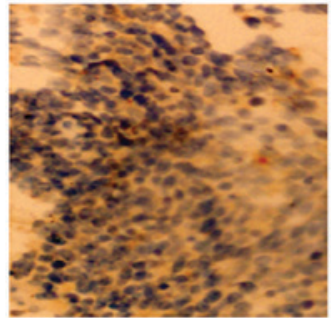

$\operatorname{ALK}(-)$

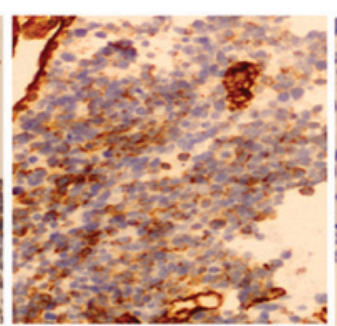

$\operatorname{VIM}(+)$

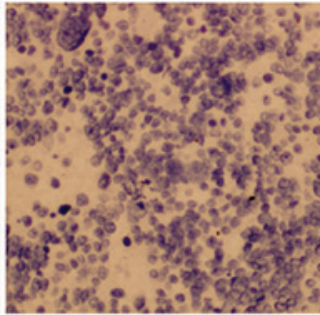

LCA (-)

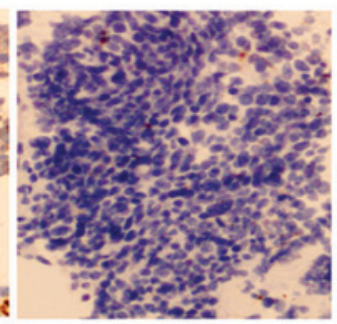

AE1/AE3 (-)

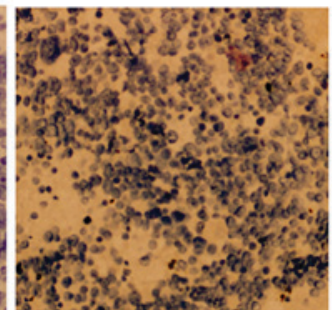

NSE (-)

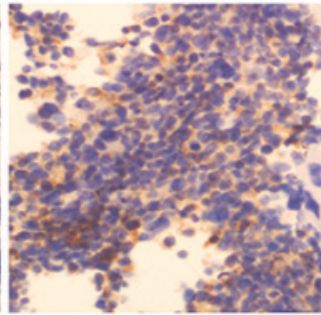

Syn (+)

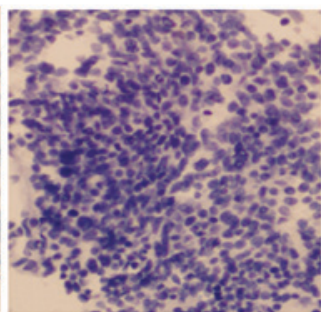

S-100 (-)

Figure 3. Immunohistochemical staining of a re-biopsy specimen of the left lower lobe mass obtained by endobronchial ultrasound transbronchial needle aspiration confirming the histological transformation to small cell lung cancer positive for the expression of TTF1, Syn and VIM. TTF1, transcription termination factor 1; NSE, neuron-specific enolase; ALK, anaplastic lymphoma kinase, Syn, synaptophysin; VIM, vimentin; LCA, leukocyte common antigen.

(4/115) (15), while other studies found that the T790M mutation is the major acquired resistance mechanism (in $40-55 \%$ of cases) after first-generation EGFR-TKI treatment $(5,16)$. A further study found that SCLC transformation occurred more frequently in lung adenocarcinomas with a deletion in EGFR exon 19 [in $50.0 \%$ (9/18) of cases examined] than in those with exon $21 \mathrm{~L} 858 \mathrm{R}$ mutation $(27.8 \%, 5 / 18)(12)$. The majority of the SCLC transformations retained the same gene mutation as the primary lung adenocarcinoma $(83.3 \%, 15 / 18)$, with only a small proportion $(16.7 \%, 3 / 18)$ losing the original gene mutation or gaining another type of gene mutation (12). This phenomenon may therefore be associated with a different mechanism of SCLC transformation.

Heterogeneity in lung cancer (including driver gene diversity and histological heterogeneity) occurs spatially and temporally during the evolution of cancer cells. The regionally separated, temporally altered driver mutations, coupled with genome instability, markedly complicates the treatment of NSCLC $(17,18)$ and is associated with an increased likelihood of postoperative relapse in patients with localized lung adenocarcinomas (19). The most common histological heterogeneity is that of adenocarcinoma mixed with squamous cell carcinoma, termed adenosquamous carcinoma (20). Combined SCLC and NSCLC histology has been reported by two large studies. The first study analyzed 176 SCLC tumors and found that 17 (9.7\%) tumors had an NSCLC component (21). The second study examined the histology of 429 SCLC tumors, finding that 9 (2.1\%) tumors contained NSCLC cells (6 adenocarcinoma and 3 squamous cell carcinoma) (22). According to these data, re-biopsy when tumors do not respond as initially expected or when the responses among two or more different lesions are discordant in advanced lung cancer may confirm a dominant histology that was not identified at the initial diagnosis. Histological transformation from NSCLC to SCLC following treatment with an EGFR-TKI has been previously reported as a rare type of histological heterogeneity (23).

The fact that the majority of transformed SCLCs retained an original EGFR-activating mutation in the study by Jiang et al (12) means that SCLC may have been transformed from primary adenocarcinoma or that the SCLC and adenocarcinoma cells originated from the same cancer stem cells as a mechanism of resistance to EGFR-TKI treatment. A small proportion of transformed SCLC cells did not have the same EGFR mutations present in the original adenocarcinomas, as in the current case report (12). These patients may have tumors with a combined adenocarcinoma and SCLC histology, which was not apparent at the time of the initial diagnosis. When the adenocarcinoma was successfully treated with an EGFR-TKI, the SCLC component became dominant as it was resistant to EGFR-TKI treatment. A prior study reported that retinoblastoma protein $(\mathrm{Rb})$ expression was lost in cases where the histological phenotype had changed from NSCLC to SCLC following EGFR-TKI therapy, whereas $\mathrm{Rb}$ was rarely lost in those that retained the NSCLC phenotype. This is considered one of the molecular mechanisms of SCLC transformation (24). Neuroendocrine differentiation can occur during the transformation of SCLC and may lead to increased chemosensitivity (25). It can therefore be concluded that SCLC cells may trans-differentiate from primary adenocarcinoma cells, originate from the minor pre-existent SCLC under the selection pressure of EGFR-TKIs, or arise from multi-potent cancer stem cells. This phenomenon emphasizes the importance of re-biopsy in the clinical design of a treatment regimen.

A rapid increase in serum NSE and a poor response to EGFR-TKIs is usually an indication of transformation from adenocarcinoma to SCLC (26). In the present case, the markedly increased serum levels of NSE highlighted the necessity of repeat biopsies. The present case indicates that patients 
could benefit from the routine testing of serum NSE levels to monitor SCLC transformation. The majority of cases of SCLC transformation have exhibited neuroendocrine differentiation with synaptophysin positive expression and responded well to initial EP chemotherapy $(12,21,27)$. The SCLC tissue in the present case was positive for synaptophysin expression, but was resistant to first-line EP chemotherapy. Immunohistochemical analysis of SCLC tissue reveals vimentin expression (28). Vimentin is a major constituent of the intermediate filament family of proteins and a biomarker of mesenchymal tissue that is overexpressed in various epithelial cancer types, including in lung cancer cells (29). Vimentin expression in lung cancer cells is an independent prognostic predictor of poor survival in primary NSCLC (30), and its expression is significantly lower in squamous cell carcinoma than in adenocarcinoma (31). Vimentin is recognized as a canonical marker for the epithelial-mesenchymal transition (32), and is associated with tumor growth and metastasis (33), serving as a potential molecular target for cancer therapy (34). Withaferin A, a small-molecule antagonist of vimentin, can elicit apoptosis, decrease angiogenesis and induce vimentin cleavage in vimentin-expressing tumor cells (35). Vimentin is also a predictive biomarker of a worse outcome from erlotinib therapy (36). In the present case, vimentin expression in SCLC was the molecular mechanism behind the resistance to chemotherapy.

In conclusions, the present study reported the case of a 74-year-old man who was initially diagnosed with lung adenocarcinoma with a deletion in exon 19 of EGFR. The patient was treated with gefitinib and relapsed after 1 year. Transformation to EGFR-exon 19 deletion-negative SCLC was the reason for gefitinib resistance. The patient was refractory to EP chemotherapy owing to vimentin expression. Transformation from adenocarcinoma to SCLC may originate from a minor pre-existent SCLC cell population under the selective pressure of EGFR-TKI treatment. NSE serum level may be useful for detecting early histological transformation, while re-biopsy is also important to EGFR-TKI-resistant patients, as it allows for genetic and histological re-evaluation of the disease.

\section{Acknowledgements}

This study was supported by the National Natural Science Foundation (grant nos. 81272586 and 81272646). The authors would like to thank Dr Fred Bogott for providing assistance with English language editing.

\section{References}

1. Siegel RL, Miller KD and Jemal A: Cancer statistics, 2015. CA Cancer J Clin 65: 5-29, 2015.

2. Wakelee $\mathrm{H}$ and Belani CP: Optimizing first-line treatment options for patients with advanced NSCLC. Oncologist 10 (Suppl 3): S1-S10, 2005.

3. Wu JY, Wu SG, Yang CH, Chang YL, Chang YC, Hsu YC, Shih JY and Yang PC: Comparison of gefitinib and erlotinib in advanced NSCLC and the effect of EGFR mutations. Lung Cancer 72: 205-212, 2011.

4. Xu M, Xie Y, Ni S and Liu H: The latest therapeutic strategies after resistance to first generation epidermal growth factor receptor tyrosine kinase inhibitors (EGFR TKIs) in patients with non-small cell lung cancer (NSCLC). Ann Transl Med 3: 96, 2015.
5. Camidge DR, Pao W and Sequist LV: Acquired resistance to TKIs in solid tumours: Learning from lung cancer. Nat Rev Clin Oncol 11: 473-481, 2014.

6. Li W, Ren S, Li J, Li A, Fan L, Li X, Zhao C, He Y, Gao G, Chen X, et al: T790M mutation is associated with better efficacy of treatment beyond progression with EGFR-TKI in advanced NSCLC patients. Lung Cancer 84: 295-300, 2014.

7. Brugger W and M Thomas: EGFR-TKI resistant non-small cell lung cancer (NSCLC): New developments and implications for future treatment. Lung Cancer 77: 2-8, 2012.

8. Cretella D, Saccani F, Quaini F, Frati C, Lagrasta C, Bonelli M, Caffarra C, Cavazzoni A, Fumarola C, Galetti M, et al: Trastuzumab emtansine is active on HER-2 overexpressing NSCLC cell lines and overcomes gefitinib resistance. Mol Cancer 13: 143, 2014.

9. Lin Y, Wang X and Jin H: EGFR-TKI resistance in NSCLC patients: Mechanisms and strategies. Am J Cancer Res 4: 411-435, 2014.

10. Li Y, Yang Z, Li W, Xu S, Wang T, Wang T, Niu M, Zhang S, Jia L and Li S: TOPK promotes lung cancer resistance to EGFR tyrosine kinase inhibitors by phosphorylating and activating c-Jun. Oncotarget 7: 6748-6764, 2016.

11. Rudisch A, Dewhurst MR, Horga LG, Kramer N, Harrer N, Dong M, van der Kuip H, Wernitznig A, Bernthaler A, Dolznig H and Sommergruber W: High EMT signature score of invasive non-small cell lung cancer (NSCLC) cells correlates with NFKB driven colony-stimulating factor 2 (CSF2/GM-CSF) secretion by neighboring stromal fibroblasts. PLoS One 10: e0124283, 2015.

12. Jiang SY, Zhao J, Wang MZ, Huo Z, Zhang J, Zhong W and $\mathrm{Xu} Y$ : Small-cell lung cancer transformation in patients with pulmonary adenocarcinoma: A case report and review of literature. Medicine (Baltimore) 95: e2752, 2016.

13. Watanabe S, Sone T, Matsui T, Yamamura K, Tani M, Okazaki A, Kurokawa K, Tambo Y, Takato H, Ohkura N, et al: Transformation to small-cell lung cancer following treatment with EGFR tyrosine kinase inhibitors in a patient with lung adenocarcinoma. Lung Cancer 82: 370-372, 2013.

14. Oser MG, Niederst MJ, Sequist LV and Engelman JA: Transformation from non-small-cell lung cancer to small-cell lung cancer: Molecular drivers and cells of origin. Lancet Oncol 16: e165-e172, 2015.

15. Yu HA, Arcila ME, Rekhtman N, Sima CS, Zakowski MF, Pao W, Kris MG, Miller VA, Ladanyi M and Riely GJ: Analysis of tumor specimens at the time of acquired resistance to EGFR-TKI therapy in 155 patients with EGFR-mutant lung cancers. Clin Cancer Res 19: 2240-2247, 2013.

16. Wu SG, Liu YN, Tsai MF, Chang YL, Yu CJ, Yang PC, Yang JC, Wen YF and Shih JY: The mechanism of acquired resistance to irreversible EGFR tyrosine kinase inhibitor-afatinib in lung adenocarcinoma patients. Oncotarget 7: 12404-12413, 2016

17. de Bruin EC, McGranahan N, Mitter R, Salm M, Wedge DC, Yates L, Jamal-Hanjani M, Shafi S, Murugaesu N, Rowan AJ, et al: Spatial and temporal diversity in genomic instability processes defines lung cancer evolution. Science 346: 251-256, 2014.

18. McGranahan N, Favero F, de Bruin EC, Birkbak NJ, Szallasi Z and Swanton C: Clonal status of actionable driver events and the timing of mutational processes in cancer evolution. Sci Transl Med 7: 283ra54, 2015.

19. Zhang J, Fujimoto J, Zhang J, Wedge DC, Song X, Zhang J, Seth S, Chow CW, Cao Y, Gumbs C, et al: Intratumor heterogeneity in localized lung adenocarcinomas delineated by multiregion sequencing. Science 346: 256-259, 2014.

20. Uramoto H, Yamada S and Hanagiri T: Clinicopathological characteristics of resected adenosquamous cell carcinoma of the lung: Risk of coexistent double cancer. J Cardiothorac Surg 5: $92,2010$.

21. Adelstein DJ, Tomashefski JF Jr, Snow NJ, Horrigan TP and Hines JD: Mixed small cell and non-small cell lung cancer. Chest 89: 699-704, 1986.

22. Mangum MD, Greco FA, Hainsworth JD, Hande KR and Johnson DH: Combined small-cell and non-small-cell lung cancer. J Clin Oncol 7: 607-612, 1989.

23. Kim WJ, Kim S, Choi H, Chang J, Shin HJ, Park CK, Oh IJ, Kim KS, Kim YC and Choi YD: Histological transformation from non-small cell to small cell lung carcinoma after treatment with epidermal growth factor receptor-tyrosine kinase inhibitor. Thorac Cancer 6: 800-804, 2015

24. Niederst MJ, Sequist LV, Poirier JT, Mermel CH, Lockerman EL, Garcia AR, Katayama R, Costa C, Ross KN, Moran T, et al: RB loss in resistant EGFR mutant lung adenocarcinomas that transform to small-cell lung cancer. Nat Commun 6: 6377, 2015. 
25. Chang Y, Kim SY, Choi YJ, So KS, Rho JK, Kim WS, Lee JC, Chung JH and Choi CM: Neuroendocrine differentiation in acquired resistance to epidermal growth factor receptor tyrosine kinase inhibitor. Tuberc Respir Dis (Seoul) 75: 95-103, 2013.

26. Zhang Y, Li XY, Tang Y, Xu Y, Guo WH, Li YC, Liu XK, Huang CY, Wang YS and Wei YQ: Rapid increase of serum neuron specific enolase level and tachyphylaxis of EGFR-tyrosine kinase inhibitor indicate small cell lung cancer transformation from EGFR positive lung adenocarcinoma? Lung Cancer 81: 302-305, 2013

27. Hwang KE, Jung JW, Oh SJ, Park MJ, Shon YJ, Choi KH, Jeong ET and Kim HR: Transformation to small cell lung cancer as an acquired resistance mechanism in EGFR-mutant lung adenocarcinoma: A case report of complete response to etoposide and cisplatin. Tumori 101: e96-e98, 2015.

28. Molenaar WM, Oosterhuis JW, Oosterhuis AM and Ramaekers FC: Mesenchymal and muscle-specific intermediate filaments (vimentin and desmin) in relation to differentiation in childhood rhabdomyosarcomas. Hum Pathol 16: 838-843, 1985.

29. Kidd ME, Shumaker DK and Ridge KM: The role of vimentin intermediate filaments in the progression of lung cancer. Am J Respir Cell Mol Biol 50: 1-6, 2014.

30. Al-Saad S, Al-Shibli K, Donnem T, Persson M, Bremnes RM and Busund LT: The prognostic impact of NF-kappaB p105, vimentin, E-cadherin and Par6 expression in epithelial and stromal compartment in non-small-cell lung cancer. Br J Cancer 99: 1476-1483, 2008.
31. Tadokoro A, Kanaji N, Liu D, Yokomise H, Haba R, Ishii T, Takagi T, Watanabe N, Kita N, Kadowaki N and Bandoh S: Vimentin regulates invasiveness and is a poor prognostic marker in non-small cell lung cancer. Anticancer Res 36: 1545-1551, 2016.

32. Liu CY, Lin HH, Tang MJ and Wang YK: Vimentin contributes to epithelial-mesenchymal transition cancer cell mechanics by mediating cytoskeletal organization and focal adhesion maturation. Oncotarget 6: 15966-15983, 2015.

33. Pan Y, Li X, Duan J, Yuan L, Fan S, Fan J, Xiaokaiti Y, Yang H, Wang Y and Li X: Enoxaparin sensitizes human non-small-cell lung carcinomas to gefitinib by inhibiting DOCK1 expression, vimentin phosphorylation and Akt activation. Mol Pharmacol 87: 378-390, 2015.

34. Satelli A and Li S: Vimentin in cancer and its potential as a molecular target for cancer therapy. Cell Mol Life Sci 68: 3033-3046, 2011.

35. Lahat G, Zhu QS, Huang KL, Wang S, Bolshakov S, Liu J, Torres K, Langley RR, Lazar AJ, Hung MC and Lev D: Vimentin is a novel anti-cancer therapeutic target; insights from in vitro and in vivo mice xenograft studies. PLoS One 5: e10105, 2010.

36. Richardson F, Young GD, Sennello R, Wolf J, Argast GM, Mercado P, Davies A, Epstein DM and Wacker B: The evaluation of E-Cadherin and vimentin as biomarkers of clinical outcomes among patients with non-small cell lung cancer treated with erlotinib as second- or third-line therapy. Anticancer Res 32: 537-552, 2012. 